\title{
Intrusion patterns in rapid serial visual presentation tasks with two response dimensions
}

\author{
JUAN BOTELLA and MARIA L. GARCIA \\ Universidad Autónoma de Madrid, Madrid, Spain \\ and \\ MARIBEL BARRIOPEDRO \\ Universidad Pontificia de Comillas, Madrid, Spain
}

\begin{abstract}
A rapid serial visual presentation (RSVP) paradigm using both one and two response dimensions was used to test parallel processing models of stimulus dimensions. Fifty subjects were asked to report the identity and/or color of a target uppercase word inserted in a series of lowercase words. The results produced a predominance of posttarget intrusions for color responses and a predominance of pretarget intrusions for identity responses. The requirement of a response to a second dimension impaired hit rates but did not change the pattern of intrusions. An examination of the distributions of intrusions in each response dimension as a function of the response given to the other dimension showed an unexpectedly high percentage of simultaneous hits, a moderate covariation between both responses, and the same patterns of intrusions when compared with the general distributions. While these results seem to be compatible with parallel models of processing for stimulus dimensions, two modifications to this model are suggested. First, the processing of response dimension(s) needs some attentional resources. Second, provision for a mixed model is indicated, which would include trials where no illusory conjunctions are formed.
\end{abstract}

Rapid serial visual presentation (RSVP) tasks have been used to study the mechanisms involved in the formation of illusory conjunctions, or incorrect combinations of features actually presented. In this type of experimental paradigm, a series of visual stimuli are presented, all in a single spatial location and at a high speed. Subjects are asked to report a specific feature of a stimulus in the series that complies with previously specified conditions (the target stimulus). Botella and Eriksen (1992) have proposed a number of terms for these paradigms: key dimension, the stimulus dimension that defines the target; key feature, the specific value that the key dimension takes in a particular trial; response dimension, the stimulus dimension that is asked of the subject; (target) response feature, the value that the target takes on that dimension (the one that if reported would be considered as a hit). Thus, for example, if colored letters are presented and subjects are asked to report the color of a specified letter, then the key dimension is identity (determined by shape) and color is the response dimension. In a trial where the subject is asked to report the color of the letter $\mathrm{T}$, and in which

Thanks are due Tram Neill and an anonymous reviewer for their constructive comments on the draft. Correspondence concerning this article should be addressed to J. Botella, Facultad de Psicologia, Universidad Autónoma de Madrid, Cantoblanco s/n, 28049 Madrid, Spain (e-mail: juanb@ccuam3.sdi.uam.es). the $T$ is presented in white, the key feature is the value $T$ and the response feature of the target is the value white.

The most interesting result in these experiments is that subjects sometimes report a feature that belongs to an item other than the target. These types of errors have been designated intrusions. The most frequent kind of intrusion has been that of reporting the response feature of the item immediately following the target. Errors consisting of reporting the response feature of an item presented after the target are called posttarget intrusions; errors consisting of reporting the response feature of an item presented before the target are called pretarget intrusions. According to McLean, D. E. Broadbent, and M. H. P. Broadbent (1983), when the results of an experiment show a predominance of posttarget (over pretarget) intrusions, it is said that a posttarget pattern has been found, whereas a predominance of pretarget intrusions is referred to as a pretarget pattern. No significant predominance of any of the two kinds of intrusions is called a symmetrical pattern.

Although the phenomenon that originally caught the attention of researchers working with this paradigm was the predominance of posttarget intrusions (Lawrence, 1971), not all published experiments have shown such predominance (see Botella \& Eriksen, 1992, for a review of RSVP experiments). To explain these results, serial and parallel models for processing of key and response dimensions have been formulated. D. E. Broadbent 
(1977) originally proposed a serial processing model of the stimulus dimensions to account for the predominance of posttarget intrusions. In that model, the response dimension of stimuli is not processed until the key feature is detected. The system then deals with the response dimension of the item present in that moment (the target item if the key feature detection has been quick enough, or a later item if detection has been too slow), applying a kind of conditional processing. Subsequent experiments using this paradigm revealed symmetrical patterns of intrusions (Gathercole \& D. E. Broadbent, 1984; McLean et al., 1983). To account for these patterns, D. E. Broadbent and his colleagues have advocated a parallel model similar to the one proposed by Keele and Neill (1978). In their model, subjects adopt either strategies of serial or parallel processing, these different strategies being revealed by the presence of different patterns of intrusions. However, Botella and Eriksen (1992) have proposed that the above research findings on posttarget and symmetrical patterns could be explained by a parallel model and that a serial model is unnecessary.

The basic ideas of parallel models concern modularity and simultaneity of representations. When a stimulus is presented, it is broken down into different features (Treisman \& Gelade, 1980) or codes (Keele \& Neill, 1978) that develop in a very autonomous and independent way. Subsequently, they are conjoined to form a single and integrated percept. There is strong convergence between these ideas and physiological and anatomical findings supporting the point of view that different components of visual information processing are segregated into largely independent parallel pathways (Livingston \& Hubel, 1987; Zeki, 1981). Usually, the clue to put features together is spatial location (Treisman, 1988). However, in RSVP tasks, space cannot be used as a correspondence map, and it is probably substituted by a kind of temporal-based clue. From the moment that a stimulus is presented, some amount of time is needed to have a representation of each feature. If the stimulus is replaced before those representations are available (as in RSVP experiments), then features from different stimuli can be simultaneously present in the system. When the key feature is detected, the system takes the representation of the most active response feature at that moment. In experimental conditions where the key feature is easy to detect (detection takes a short average time) and the processing of the response dimension takes a long average time, then the most active response features will come, on the average, from items presented earlier than if a response dimension that is easier to process (shorter average time) is used. Different combinations of average times to develop representations of the key and response dimensions (relative to a specific presentation rate) can produce patterns of predominance of pretarget or posttarget intrusions, or a balance between them. Simultaneity of representations must be the crucial point for feature integration in RSVP tasks.

The simple versions of parallel models described above, however, cannot account for some experimental results.
For example, those models predict a relative increase in posttarget intrusions as a consequence of increasing the average time needed to extract the key feature. However, in experiments with target-specified and target-categorized conditions, it has been shown that if the target is defined by shape, the average origin of intrusions is later than if it is defined by alphanumeric category (Gathercole \& D. E. Broadbent, 1984; McLean et al., 1983). This is contrary to the well-known fact that categorization takes more time than does identification (Dick, 1971). Botella (1992), following the suggestion of McLean et al. (1983) that perhaps the extraction of the dimensions cannot be carried out in a completely automatic way, has suggested that differential resources are allocated to the various dimensions. From his point of view, the detection of the key feature is more resource-consuming in targetcategorized conditions than in target-specified conditions. Consequently, there is a decrease in the response dimension processing rate, not in the key dimension processing rate. The predicted effect of a slowing of the key dimension processing is an earlier average origin of responses, exactly what has been observed in all published experiments with such experimental conditions. However, more empirical evidence is needed to accept this added postulate about the resource-consuming nature of dimension processing.

In the present experiment, two response dimensions were included. Lists of colored words in lowercase were presented, with one uppercase word inserted in the middle of the list (the target word). The subjects, divided into four groups, were asked to report the identity and/or the color of the target. This experimental design tested the proposition that dimension processing is resourceconsuming. Accordingly, it was predicted that the requirement of response to a second dimension would have an effect on the response to the first one. In addition, the present experiment explored the parallel model outlined above, via the examination of conditional patterns of intrusions-that is, the frequencies of intrusions in one response to a dimension as a function of the response given to the other. From a parallel model, it was predicted that the two response dimensions will not always come from the same stimulus, but there would be a moderate covariation between their origins. Specifically, in trials where the detection of the key feature takes an unusually short interval, earlier average origins would be observed for both response dimensions than in trials where the key feature detection takes an unusually long time. Given that in the three amounts of time indicated (extraction of key feature and of both response features) there is some variability, the expected relationship will be of moderate intensity. Although detection time cannot be directly observed, some specific predictions can be derived from the conditional distributions. For example, if we take those trials with a -1 intrusion in one response dimension (e.g., identity), the average origin for responses in the other response dimension (e.g., color) would be earlier than for those trials with a +1 intrusion in the identity response. 


\section{METHOD}

Subjects

Fifty undergraduate students at Universidad Autónoma of Madrid, with normal or corrected-to-normal vision, served as volunteers for this experiment.

\section{Stimuli and Material}

Lists of 13 words were constructed, using the 400 most often used Spanish nouns with lengths of four, five, or six letters (see Juilland \& Chang Rodriguez, 1964). Word length was held as constant as possible across word lists, and no word was repeated in the same list. Words were presented in lowercase with the exception of the target word, which was presented in uppercase. The target word was randomly presented in the sixth, seventh, or eighth position in the word list. Items on each list are referred to according to their position relative to the target. Thus, the word presented just before the target is Item -1 , the word previous to this is Item -2 , and so on. Words presented after the target are Items $+1,+2$, +3 , and so on. The target word, the two words presented before it, and the two presented after it are referred to as the critical set.

Words were in five colors: blue, green, red, yellow, and white (the computer standard colors). The assignment of colors to words was as follows: (1) a random color was selected for the target word (the target word was in each of the five colors with the same frequency; that color was never repeated in the same list); (2) the other four colors were also assigned randomly, without replacement, to the other four words in the critical set; and (3) once the critical set was prepared, colors were assigned randomly to the rest of the list using the same four colors that were used for the four items nearest the target, but with the restriction that two consecutive words never shared the same color.

The presentation of word lists and the response collection were carried out by an Inves PC-XT microcomputer. The program was elaborated using MEL (Schneider, 1988).

\section{Procedure}

Four groups, which were presented the same material, were created on the basis of the order of task administration and the number of response dimensions. The experimental conditions are labeled as follows: $I C$, the target identity was asked first, followed by color; $C I$, color was asked first, followed by target identity; $I$, only the identity was asked for; and $C$, only the color was asked for. These conditions affected only the response collection, but not the stimuli presentation, which remained constant. Each trial started with six black asterisks (fixation) on the screen, which appeared in the same location where the word series would appear. The screen background was constantly dark gray. When the subject was ready, he/she pressed the keyboard space bar. The fixation remained for a variable interval ranging from 500 to $666 \mathrm{msec}$ (average $583 \mathrm{msec}$ ). The fixation was replaced by the first word of the series, the first word was replaced by the second, and so on, until the last word. Each word was presented for $83 \mathrm{msec}$ and was replaced immediately by the next word, without any intervening period. That is, the exposure interval equaled the stimulus onset anychrony (SOA). The last word was replaced again by the six asterisks (the mask) during $83 \mathrm{msec}$, to avoid the tendency of the subjects to report the last word in the list. The mask was then replaced by the first response menu.

The identities menu contained six numbered words, corresponding to the five words of the critical set plus one word not presented in the list, to assess for possible random responses. The six words were in black lowercase and in a random order (different from the order of presentation in the series). Two additional options, "I don't know" and "not in the menu," were also included. Presumably, trials with "not in the menu" responses correspond mainly to trials where the subject would have selected a word more than two positions away from the target if it had been included in the menu. The subjects responded by pressing the key corresponding to the number of the selected option.

The colors menu had six numbered alternatives. The first five were the names of the five colors used for the words, each name in its own designated color, and in the same order for all trials. The sixth option was "I don't know." After the subject responded to the second menu, the screen was deleted and the fixation was presented again until the subject pressed the space bar to begin the next trial. Figure 1 shows a diagram of the procedure. The only difference between the IC and CI programs was the order in which the menus were presented after each list. In the programs of I and $\mathrm{C}$ tasks, only one of the menus was presented (identity or color, respectively).

The same stimuli, 88 different word lists, were used in each of the four programs. In 80 lists, the procedure described above was used; in the other 8 lists, the target word was presented in lowercase. These trials were designed to control for random responses (see Botella \& Eriksen, 1992). Furthermore, another group of 20 similar lists were constructed to be applied at the beginning of the session to familiarize the subjects with the task and the procedure. Groups 1 and 2 included 10 subjects each. Group 1 first performed Task I and then performed Task C; Group 2 first performed Task $C$ and then Task I. Group 3 and 4 included 15 subjects each. Group 3 first performed the IC task and then the CI task; Group 4 performed those tasks in the reverse order. Sessions lasted about $40 \mathrm{~min}$.

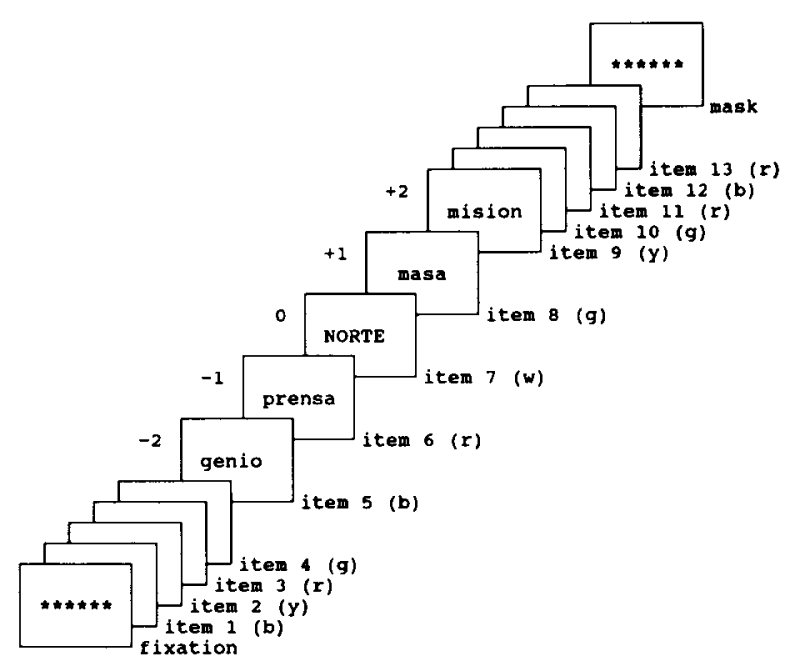

IDENTITIES MENU

COLORS MENU
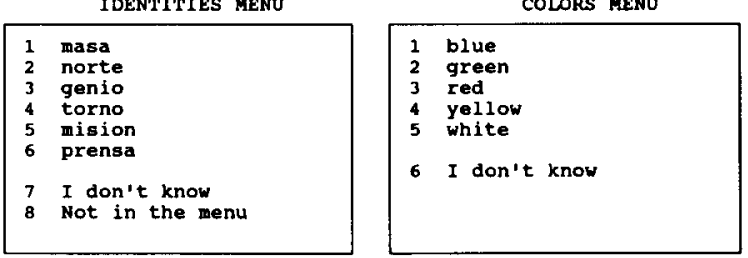

Figure 1. Representation of a typical trial. Items 5-9 comprise the critical set in this trial, and the target word is norte. The word not presented in the list is torno; number 4 in the identities menu. All identities menus were presented in black in all trials. In the colors menus, each option (color name) was presented in the designated color, and the option "I don't know" was presented in black. In this figure, the colors are indicated as follows: $b=$ blue, $g=$ green, $r=$ red, $y=$ yellow, and $w=$ white. The words used in this particular trial are masa (mass), norte (north), genio (genius), torno (winch), mision (mission), and prensa (press). 
Responses were classified according to the position of the item, with the response feature selected by the subject in the menu. Thus, for example, if in the trial of Figure 1 the subject selects the first alternative in the identities menu, it would be classified as a +1 intrusion; if the second is selected, it would be classified as a hit. On the other hand, the third option in the colors menu would be classified as an intrusion from -1 position, and the fourth option would be classified as an intrusion from +2 position.

\section{RESULTS}

The following results are divided into two sections. The first describes preliminary analyses, which were conducted to assess and eliminate "order" as a confounding variable. The second section presented the analyses used to test the primary hypotheses of this experiment. For both types of analyses, the presence of significant patterns of intrusions are checked by the sign test, taking the number of subjects showing a predominance of one kind of intrusions and testing the null hypothesis that the intrusions were randomly distributed (in statistical terms, that any subject has the same probability of showing a predominance of pretarget versus posttarget intrusions).

\section{Order Effects}

In the present experiment, order effects could show up for tasks and/or response dimensions. Results obtained in tasks carried out first were compared with the results obtained in the same tasks carried out second. Performance in single-response tasks was impaired when it was carried out second. Hits on identity were $68.2 \%$ for Group 1 and $67.8 \%$ for Group 2; hits on color responses were $67.3 \%$ for Group 2 and $54.6 \%$ for Group 1 . However, in two-response tasks, performance improved when the task was carried out second. Hits on identity in the IC task were $56.1 \%$ for Group 3 and $58.3 \%$ for Group 4; hits on color responses were $42.6 \%$ for Group 3 and $52.4 \%$ for Group 4 . Identity hits for the CI task were $53.3 \%$ for Group 4 and $61.7 \%$ for Group 3; color response hits were $47.3 \%$ for Group 4 and $52.1 \%$ for Group 3. These differences were not statistically significant. No order effects were found for response dimensions in two-response tasks.

Given that the different tasks and responses showed the same pattern of predominance for intrusion errors independently of order, we collapsed the results across groups. The distributions of responses appear in Table 1

Table 1

Percentages of Identity Responses as a Function of Response Requirements and Location Relative to Target

\begin{tabular}{ccccccc} 
& \multicolumn{6}{c}{ Location Relative to Target } \\
\cline { 2 - 6 } Requirement & -2 & -1 & $T$ & +1 & +2 & $+1-$ \\
\hline I & 6.4 & 11.2 & 68.0 & 8.3 & 6.1 & 0.82 \\
CI & 8.4 & 14.5 & 57.6 & 11.8 & 7.6 & 0.85 \\
IC & 8.3 & 14.6 & 57.2 & 11.2 & 8.7 & 0.87 \\
\hline
\end{tabular}

Note $-I=$ identity alone; $\mathrm{CI}=$ color, then identity; $\mathrm{IC}=$ identity, then color; $T=$ target. The last column $(+/-)$ shows the ratio between the number of posttarget and pretarget intrusions.
Table 2

Percentages of Color Responses as a Function of Response Requirements and Location Relative to Target

\begin{tabular}{crrrrrr}
\hline & \multicolumn{5}{c}{ Location Relative to Target } \\
\cline { 2 - 6 } Requirement & -2 & \multicolumn{1}{c}{-1} & \multicolumn{1}{c}{$\mathrm{T}$} & +1 & +2 & $+1-$ \\
\hline C & 6.4 & 5.5 & 60.9 & 14.9 & 12.2 & 2.28 \\
CI & 8.7 & 8.8 & 49.7 & 19.9 & 13.0 & 1.88 \\
IC & 10.0 & 10.5 & 47.9 & 18.2 & 13.4 & 1.54 \\
\hline
\end{tabular}

Note $-\mathrm{C}=$ color alone; $\mathrm{CI}=$ color, then identity; $\mathrm{IC}=$ identity, then color; $\mathbf{T}=$ target. The last column $(+/-)$ shows the ratio between the number of posttarget and pretarget intrusions.

and 2. Identity responses show a statistically significant predominance of pretarget intrusions in all cases (for the I task, 13/18, $p<.05$; for the IC task, 21/29, $p<.01$; for the CI task, 21/28, $p<.01$ ). For color responses, a statistically significant predominance of posttarget intrusions emerged (for the $C$ task, 19/20, $p<.0001$; for the IC task, 24/29, $p<.001$; for the CI task, 29/30, $p<.001$ )

To summarize, although some order effects showed up at a descriptive level in performance (hit rates), the patterns of intrusions did not seem to be affected by the task order or by the order of questions in tasks with two response dimensions. Identity responses showed a stable predominance of pretarget intrusions; color responses showed a systematic predominance of posttarget intrusions.

\section{Main Analysis}

The analysis presented below addresses the primary objectives of this study. The effects of the second response dimension are clear. The inclusion of a second response dimension impaired performance on the first. Thus, in identity responses, there were more hits when asked alone (68.0\% in the I Task) than when a second question about the color was included (57.2\% in IC task). This difference was statistically significant with the Mann-Whitney test $(z=2.565, p<.01)$. Similarly, in color responses, there were more hits when color alone was asked $(60.9 \%$ in the $\mathrm{C}$ task) than when a second question about the identity was added (49.7\% in CI task). Again, the difference, with the Mann-Whitney test, was found to be statistically significant $(z=3.228, p<.001)$.

Tables 3 and 4 show the patterns of intrusions in responses to one dimension as a function of the response given in the other dimension. We have taken only those trials with $-1,+1$, and hit responses in the first dimension, because the small number of -2 and +2 responses produced too many zero values. Specifically, Table 3 shows the distributions of color responses in the IC task as a function of the identity reported. Table 4 shows the distributions of identity responses in the $\mathrm{CI}$ task as a function of the color reported.

It is clear that responses in one dimension were not independent of responses given in the other, although there was not a perfect covariation. If detection of the key feature yields a kind of gating for an integral stimulus, then 
Table 3

Percentages of Color Responses in IC Task as a Function of Identity Responses

\begin{tabular}{crrrrrr}
\hline \multirow{2}{*}{$\begin{array}{c}\text { Identity } \\
\text { Responses }\end{array}$} & \multicolumn{6}{c}{ Color Responses } \\
\hline-1 & -2 & -1 & \multicolumn{1}{c}{$\mathrm{T}$} & +1 & +2 & $+1-$ \\
\hline $\mathrm{T}$ & 22.0 & 16.5 & 11.0 & 29.0 & 21.5 & 1.31 \\
+1 & 4.0 & 5.8 & 71.9 & 11.3 & 7.1 & 1.88 \\
\hline
\end{tabular}

Note $-\mathrm{T}=$ target. The last column $(+/-)$ shows the ratio between the number of posttarget and pretarget intrusions.

Table 4

Percentages of Identity Responses in CI Task as a Function of Color Responses

\begin{tabular}{crrrrrr}
\hline \multirow{7}{*}{ Color } & \multicolumn{7}{c}{ Identity Responses } \\
\cline { 2 - 6 } Responses & \multicolumn{1}{c}{-2} & \multicolumn{1}{c}{-1} & \multicolumn{1}{c}{$\mathrm{T}$} & \multicolumn{1}{c}{+1} & +2 & $+1-$ \\
\hline-1 & 24.4 & 30.7 & 24.4 & 10.2 & 10.2 & 0.37 \\
$\mathrm{~T}$ & 3.8 & 7.6 & 79.6 & 5.6 & 3.3 & 0.78 \\
+1 & 13.6 & 23.5 & 39.3 & 16.7 & 6.8 & 0.63 \\
\hline
\end{tabular}

Note-T $=$ target. The last column $(+/-)$ shows the ratio between the number of posttarget and pretarget intrusions.

the origin of the two response dimensions would always be the same, its being the target or any other stimulus. On the contrary, the pattern in the total results was maintained in the conditional distributions, although to a different degree. Thus, in the color responses of the IC task shown in Table 3, a systematic predominance of posttarget intrusions was observed for each identity response (for -1 responses, 17/24, $p<.05$; for hit responses, 22/28, $p<.01$; for +1 responses, $23 / 27, p<.001$ ). However, the ratio of posttarget to pretarget intrusions increased, the later the identity was reported. That relationship was moderate but clear, as we expected.

Identity responses in the CI task showed (see Table 4) a systematic predominance of pretarget intrusions for each color response (for -1 responses, 19/23, $p<.001$; for hit responses, 19/27, $p<.05$; for +1 responses, 20/24, $p<.001$ ). This predominance of pretarget intrusions was larger when a pretarget intrusion had been given in color. It was not the case that the pattern of intrusions with a hit in the first dimension was an intermediate between the other two. The distributions of Tables 3 and 4 clearly show that when there is a mistake in the first dimension, especially a -1 intrusion, performance is impaired dramatically in the second.

\section{DISCUSSION}

Overall, the results of the present experiment correspond with parallel processing models, although some modifications are proposed. Prior to addressing the main hypotheses related to these models, however, the presence of order effects (either of tasks or of questions) as a possible explanation of the obtained results were explored. It seems that in single-response tasks, performance tends to be slightly worse when the task is performed second, rather than first. However, in two-response tasks, the difference is the opposite: performance improves when carried out second. The interpretation of these effects is not completely clear. It seems that, in single-response tasks, the previous administration of the other task yields some interference, probably due to the fact that the effect of practice on attention focusing in the other dimension cannot be immediately eliminated. The results from single-response tasks suggest a kind of varied mapping (Schneider \& Shiffrin, 1977; Shiffrin \& Schneider, 1977). However, with two response dimensions, there is an opposite effect, that is, it seems that there is enhanced performance in the task carried out second. Perhaps the reason for this reversal is that, for this second set of tasks, there is a consistent mapping, because attention must be focused on both response dimensions simultaneously. This explanation, however, would not apply to patterns of intrusion, because no order effects were found. Color responses showed a predominance of posttarget intrusions in all tasks studied (C, IC, and CI), and identity responses showed a pretarget predominance in all the tasks studied (I, IC, and CI).

The effect of including a second response dimension appears to influence responses to the first. Performance on identity responses was influenced by the color question (comparison between the I task and the IC task), and the same happened to performance on color responses when a second question about the identity was included (comparison between the $\mathrm{C}$ task and the $\mathrm{CI}$ task). However, the patterns of intrusions were not influenced by this manipulation; they remained pretarget for identity and posttarget for color. According to the resource-consuming postulate described in the introduction section, the inclusion of a second response dimension could yield a partial withdrawal of resources allocated to the processing of the key dimension and the first response dimension. Monte Carlo simulations show that the result of a general slowing in the processing of the three dimensions could simply be an impairment on hits, while the pattern of intrusions remains unchanged (Botella \& Villar, 1989). The present results would support the need for such a postulate and provide convergent evidence for Botella's (1992) interpretation of the different patterns found in target-specified and target-categorized conditions.

The double-response conditions were also included to explore the parallel model, because a covariation between the origins of the responses in both dimensions could cast light on the processes that occur in this kind of task. If key feature detection gives way to integral stimuli representations, then the two response dimensions would show a perfect covariation. Our results fail to support this prediction. However, a complete independence would also be incompatible with the ideas of parallel models. The distributions of the conditions in Tables 3 and 4 are an intermediate between those extreme points and, thus, support the parallel model outlined in the introduction.

In contrast to the parallel model, however, our results show a higher than expected percentage of hits in the second dimension when there is a hit in the first one. It seems 
that, in double hits, there is more than temporal coincidence. The response-time results of Botella (1992) suggest that processing in trials resulting in a hit can be different from those resulting in an intrusion. Botella and Villar (1989) also found that, in order to have a good fit between experimental data and Monte Carlo simulations with the parallel models, additional trials with a correct response was needed. This suggestion was followed by Botella (1992), who proposed that there is some number of trials where subjects are efficient (and quick) enough to build a complete representation of the target stimulus before it is replaced by the next item. In the rest of the trials, they make a kind of "reconstruction" of the target, making inferences about the simultaneity of past items on the basis of the strength of the traces of the stimulus features. When some proportion of trials with correct responses is added to a set of trials developed according to the postulates of parallel models, many of the published experimental results fit nicely with that mixed model. Among them, for example, the surprisingly high number of correct responses in many experiments, the unexpected level of coincidence of hits in the two response dimensions of the present experiment, and a lower mean response time for hits in the experiment by Botella (1992).

In addition to the previous interpretations, the results of the present study are connected with the conclusions of Botella and Villar (1989) and Botella and Eriksen (1991), in that the presence of different patterns of intrusion cannot be used as an argument to make inferences about potential processing strategies (e.g., serial strategies from posttarget predominance patterns and parallel strategies from symmetrical and pretarget patterns). Neither of these processing strategies can be inferred from the double-response conditions in the present experiment (or both simultaneously!).

Another interesting result that appears to contradict the literature is the pretarget pattern observed in the identity responses. In the original experiment of Lawrence (1971) and in later experiments (Botella \& Villar, 1986), the identification of the word that matches a simple criterion (physical) has shown posttarget patterns. From the parallel model outlined earlier, it is clear that the patterns of intrusions are not inherent to the response dimensions. The same response dimension can show different patterns when combined with different key dimensions when the presentation rate is changed or when it is facilitated by clearness of stimuli used.

In conclusion, the addition of a second response dimension may be useful in shedding light on the processes involved in RSVP tasks. Moreover, this type of paradigm can be helpful in studying the relative average times needed to extract different response dimensions. While the results obtained in this experiment are in general agreement with parallel processing models, such models probably require some modifications-among them, the inclusion of two postulates, one addressing the consumption of processing resources to extract the stimulus dimensions, and the other addressing the inclusion of a percentage of trials which systematically produce correct responses.

\section{REFERENCES}

BotelLA, J. (1992). Target-specified and target-categorized conditions in RSVP tasks as reflected by detection time. Bulletin of the Psychonomic Society, 30, 197-200.

Botella, J., \& ERIKsen, C. W. (1991). Pattern changes in rapid serial visual presentation tasks without strategic shifts. Bulletin of the Psychonomic Society, 29, 105-108.

Botella, J., \& ERIKSEN, C. W. (1992). Filtering versus parallel processing in RSVP tasks. Perception \& Psychophysics, 51, 334-343.

Botella, J., \&illar, M. V. (1986). Identificación de palabras en presentación rápida de series visuales: Una réplica del "efecto Lawrence." Conocimiento y Acción, 1, 7-21.

Botella, J., \& Villar, M. V. (1989). Modelos de procesamiento en tareas de presentación rápida de series visuales. Psicológica, 10, 179-203.

Broadbent, D. E. (1977). The hidden preattentive processes. American Psychologist, 32, 109-118.

Dick, A. O. (1971). Processing time for naming and categorization of letters and numbers. Perception \& Psychophysics, 9, 350-352.

Gathercole, S. E., Broadbent, D. E. (1984). Combining attributes in specified and categorized target search: Further evidence for strategic differences. Memory \& Cognition, 12, 329-337.

Juilland, A., \& Chang Rodriguez, E. (1964). Frequency dictionary of Spanish words. London: Mouton.

KeEle, S. W., NeILl, W. T. (1978). Mechanisms of attention. In E. C. Carterette \& M. P. Friedman (Eds.), Handbook of perception (Vol. 9, pp. 3-47). New York: Academic Press.

LAWRENCE, D. H. (1971). Two studies of visual search for word targets with controlled rates of presentation. Perception \& Psychophysics, 10, 85-89.

Livingston, M. S., Hubel, D. H. (1987). Psychophysical evidence for separate channels for the perception of form, color, movement and depth. Joumal of Neuroscience, 7, 3416-3468.

Mclean, J. P., Broadbent, D. E., \& Broadbent, M. H. P. (1983). Combining attributes in rapid sequential visual presentation tasks. Quarterly Joumal of Experimental Psychology, 22, 279-287.

SCHNEIDER, W. (1988). Micro Experimental Laboratory: An integrated system for IBM PC compatibles. Behavior, Research, Methods, Instruments, \& Computers, 20, 206-217.

SCHNEIDER, W., ShIFFrin, R. M. (1977). Controlled and automatic human information processing: I. Detection, search, and attention. Psychological Review, 84, 1-67.

Shiffrin, R. M., SCHNEIDER, W. (1977). Controlled and automatic human information processing: II. Perceptual learning, automatic attending, and a general theory. Psychological Review, 84, 127-190.

Treisman, A. (1988). Features and objects: The Fourteenth Bartlett Memorial Lecture. Quarterly Journal of Experimental Psychology, 40A, 201-237.

Treisman, A., Gelade, G. (1980). A feature integration theory of attention. Cognitive Psychology, 14, 107-141.

ZEKI, S. M. (1981). The mapping of visual function in the cerebral cortex. In Y. Katsuki, R. Norgren, \& M. Sato (Eds.), Brain mechanisms of sensation. New York: Wiley.

(Manuscript received January 15, 1992; revision accepted for publication May $11,1992$. 